\title{
COMMISSIONING AND INVESTIGATION OF BEAM DYNAMICS OF PHASE I INSERTION DEVICES AT DIAMOND
}

\author{
B. Singh, R. Bartolini, R. Fielder, E.C. Longhi and I.P.S. Martin \\ Diamond Light Source, Oxfordshire, UK
}

\begin{abstract}
Diamond is a $3 \mathrm{GeV}$ low emittance third generation light source recently commissioned in Oxfordshire, UK. During Phase I of the project, seven insertion devices (IDs) have been installed and commissioned: these include 5 in-vacuum permanent magnet undulators, a variable polarization APPLE-II helical device (HU64) and a superconducting wiggler (SCW). We present our experiences commissioning these devices and the results of the investigations of their effects on beam dynamics, including orbit distortion, linear tune shifts, beta-beating and beam lifetime. Alpha-matching with local and global tune compensations, as well as the LOCO algorithm, have been used to compensate the linear optic perturbations. The results are discussed and compared with theoretical predictions. Injection with IDs in operation has also been investigated in view of future top-up operation.
\end{abstract}

\section{INTRODUCTION}

The Diamond storage ring was commissioned in 2006 [1]. It is operating at the design tune point $\left(\mathrm{Q}_{\mathrm{x}}=27.22\right.$, $\mathrm{Q}_{\mathrm{y}}=12.36$ ) of a low emittance lattice. The beam lifetime is about $20 \mathrm{~h}$ at $125 \mathrm{~mA}$ stored current in $2 / 3$ fill mode with $1 \%$ coupling and $2 \mathrm{MV}$ of accelerating voltage.

Seven Phase-I insertion devices [2] were installed before full energy commissioning began in Sep. 2006 and an $8^{\text {th }}$ in-vacuum undulator was installed in the Dec./Jan. shutdown [3]. An intensive campaign of measurements has been undertaken to characterise and correct the effect of the IDs on the beam dynamics. In this paper we describe the results of the analysis of the IDs on closed orbit correction, compensation of the linear optics perturbation, as well as on lifetime and injection efficiency. These studies have shown that the effects of the Phase I IDs on beam dynamics are mainly due to the wiggler. All installed IDs are now used routinely for user operation and further beamline commissioning.

\section{EFFECT ON CLOSED ORBIT}

The change in closed orbit as a function of gap is of the order $20-25 \mu \mathrm{m}$ rms for the in-vacuum undulators, and 50 $\mu \mathrm{m}$ for HU64 (see fig.1). Trim coil settings have been determined as a function of gap (and phase where required) and are now read automatically from feed forward tables. The residual orbit disturbance is about 1-5 $\mu \mathrm{m}$ rms. The SCW currents have also been adjusted to minimise the effect at full field (with a residual orbit of 18 $\mu \mathrm{m} \mathrm{rms}$ in horizontal and $160 \mu \mathrm{m}$ rms in vertical), while the ramp-up of the wiggler field has been corrected using

02 Synchrotron Light Sources and FELs slow orbit feedback to keep the orbit well within the beam position interlock threshold of $\pm 1 \mathrm{~mm}$..
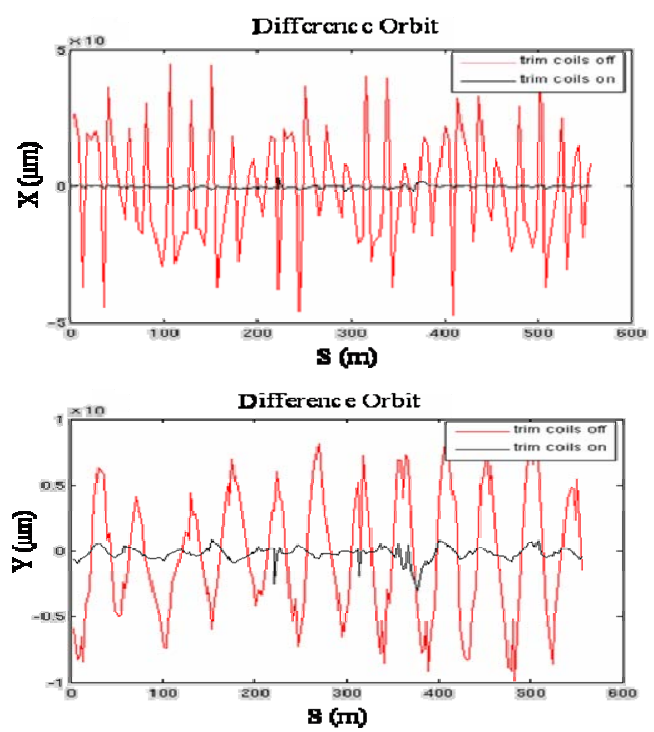

Figure 1: Difference in closed orbit for maximum and minimum gap of HU64 with/without trim coils.

\section{LINEAR OPTICS INVESTIGATIONS}

Systematic beam dynamics investigations were made to quantify the linear optic perturbation induced by the IDs and to test various schemes to compensate them [4].

The linear tuneshifts induced by the in-vacuum undulators and HU64 appear to be negligible, while the SCW generates a total vertical tuneshift of 0.013 , in very good agreement with the model prediction (see Fig. 2).

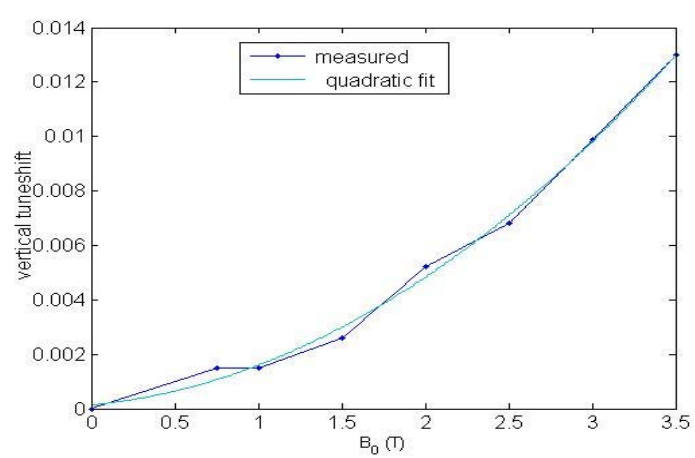

Figure 2: Vertical linear tuneshift induced by SCW.

The computer program LOCO [5,6] has been used to estimate the $\beta$-beating generated by the IDs. Prior to any ID analysis, we corrected the machine optics without any IDs closed using LOCO and the residual $\beta$-beating is less than $1 \%$. Then each ID was considered separately and LOCO measurements were performed in order to 
determine the induced $\beta$-beating. The machine model used in LOCO includes the linear focussing of the relevant ID. Only the SCW and the HU64 generated significant optics perturbations and will be reported here.

Concerning the SCW, the measured $\beta$-beating is reported in Fig. 3. The $10 \%$ variation induced in the vertical $\beta$ function is in very good agreement with the model of the wiggler with horizontal focussing $\mathrm{k}_{\mathrm{x}}=0$. The horizontal $\beta$-beating is negligible. The tune-shifts produced are $\Delta \mathrm{Q}_{\mathrm{x}} \approx 0.0, \Delta \mathrm{Q}_{\mathrm{y}}=0.013$. These measured optics perturbations due to the wiggler are consistent with the theoretical predictions made using kick maps [4].

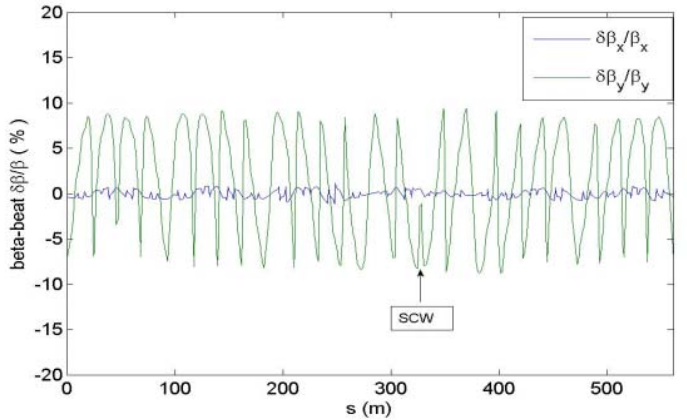

Figure 3: $\beta$-beating calculated from calibrated model of machine in presence of wiggler SCW with $\mathrm{B}_{0}=3.5 \mathrm{~T}$

The linear tune shift induced by HU64 is less than 0.004 in all polarisation modes. The induced $\beta$-beating was measured for horizontal and vertical planar polarization and was found to be about 2-3\% which is also in agreement with the prediction of the models. More studies are planned when the second APPLE-II module is installed in the machine.

Given the measured optics perturbations due to the insertion devices, we decided to attempt optics compensation only for SCW based on alpha-matching techniques and LOCO correction algorithms.

Alpha-matching: The alpha-matching technique was used to generate feed-forward tables extracted from the kick maps model of the wiggler for different levels of SCW peak field. Two pairs of quadrupoles adjacent to the $\mathrm{SCW}$, originally belonging to the Q2B and Q3B families, were used. Furthermore, a global compensation of both linear tunes was performed with the Q2D and Q3D quadrupoles families. Betatron tunes and optics function measurements with LOCO were performed with the SCW at its peak field of $3.5 \mathrm{~T}$. The residual $\beta$-beating after correction is 2-3\%, except at the SCW location (see Fig. 4 ), and the linear betatron tunes are restored. Alpha matching with local $\mathrm{Q}_{\mathrm{y}}$ compensation produced equivalent results.

LOCO algorithm: The LOCO algorithm was applied to compensate optic perturbations of the IDs in the storage ring. All the 240 quadrupoles have been used as independent variables and the convergence of the algorithm has been quite slow. Due to a technical problem with the SCW this method could only be tested for a $\mathrm{SCW}$ peak field of $\mathrm{B}_{0}=2.5 \mathrm{~T}$. The quadrupole changes 02 Synchrotron Light Sources and FELs required by $\mathrm{LOCO}$ to compensate the optic perturbation are shown in Fig. 5. The maximum quadrupole variation is about $4.5 \%$ and the changes are concentrated in a few quadrupoles in the neighbourhood of SCW, while a few distant quadrupoles change by $1 \%$. The residual $\beta$-beating measured after the $2^{\text {nd }}$ correction is shown in Fig. 6 . The optics is well corrected except in the neighbourhood of the SCW.

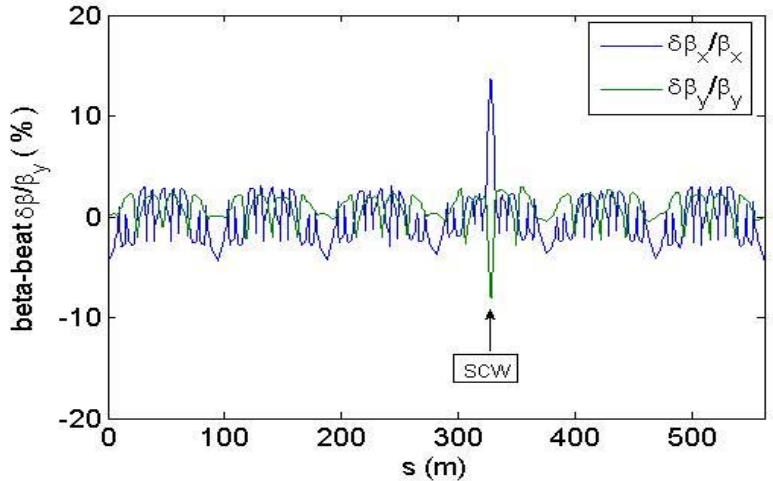

Figure 4: $\beta$-beating after alpha-matching with global tunes compensation.

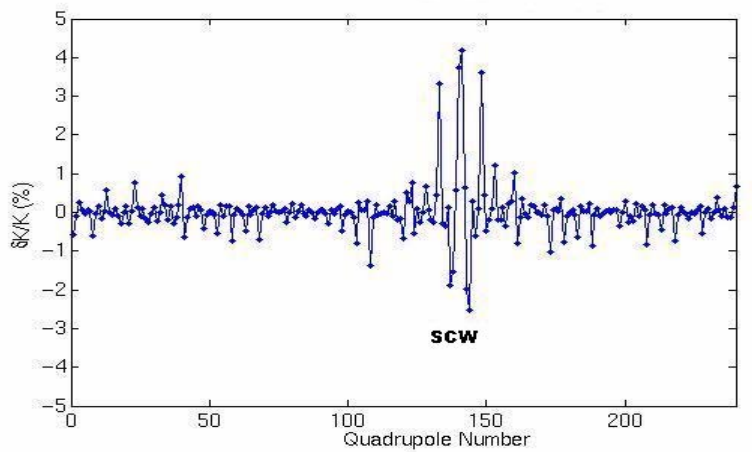

Figure 5: Relative quadrupole variations required for compensation of linear ontic perturbation of SCW.

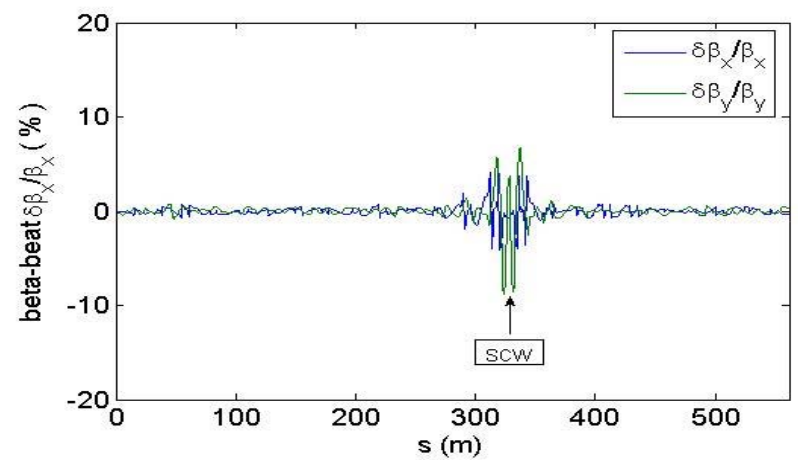

Figure 6: $\beta$-beating after $2^{\text {nd }}$ correction of the effect of the SCW with LOCO algorithm

\section{EFFECT OF IDS ON LIFETIME}

The effect of IDs on the beam lifetime was analysed during commissioning. No significant effects of the invacuum undulators $(\mathrm{gap}=7 \mathrm{~mm})$ on beam lifetime have been observed except for a slight detrimental effect of a single in-vacuum device in straight 2 , which is currently under investigation. No noticeable effect on beam lifetime due to HU64 (gap=16mm) in any of its different polarization modes has been found.

A05 Synchrotron Radiation Facilities 
Powering the SCW at maximum field without any correction reduced the beam lifetime by $10-15 \%$. However, optics compensations using either alphamatching or the loco algorithm had no impact on the beam lifetime. A TRACY-II [7] simulation of lifetime also predicts no difference between them. So, it was concluded that the reduction in lifetime is not due to optics distortions, but instead to the shrinkage of the RF energy acceptance due to additional energy loss per turn. This increased loss is given by the formula [8]:

$$
\Delta U_{I D}=\Delta U_{0}\left[1+\frac{L_{I D}}{4 \pi \rho_{0}}\left(\frac{\rho_{0}}{\rho}\right)^{2}\right]
$$

Where $\Delta \mathrm{U}_{\mathrm{ID}}$ and $\Delta \mathrm{U}_{0}$ are energy loss per turn by an electron in an ID and dipoles respectively in the ring, $\rho$ and $\rho_{0}$ are radius of curvatures of peak magnetic field of an ID and the dipoles respectively and $\mathrm{L}_{\mathrm{ID}}$ is the length of the ID. For the SCW $\Delta \mathrm{U}_{\mathrm{ID}}=0.1048 \mathrm{MeV}$ and the RF energy acceptance is reduced from $3 \%$ to $2.75 \%$ for a cavity voltage of $2 \mathrm{MV}$. This has been verified experimentally and compensated for by proportionally adjusting the cavity voltage.

\section{INJECTION STUDIES WITH IDS CLOSED FOR TOP-UP OPERATION}

In view of the planned Top-Up operation, experiments have been conducted to measure the effect of the IDs on the injection efficiency. No reduction in injection efficiency was observed for all in-vacuum IDs closed to $7 \mathrm{~mm}$ gap and HU64 closed to $16 \mathrm{~mm}$ gap. Early trials of injection with SCW at $3.5 \mathrm{~T}$ also did not show any large effect on injection efficiency ( 2\%).

The Diamond storage ring contains one horizontal and one vertical collimator in the injection straight, and tests have also been made to determine the effectiveness of using these collimators as the defining apertures of the ring with and without IDs. The aim of these tests was to determine if they can be used to concentrate particle losses to the injection region in order to provide machine and personnel protection.

In the case of a well-corrected orbit and high initial injection efficiency, the use of collimators to localise losses was shown to have limited benefit. Whilst the collimators can be used effectively for machine protection purposes (see figure 7), this comes at the expense of either a reduction in lifetime or injection efficiency.

Closing the vertical collimators to $\pm 2.5 \mathrm{~mm}$ effectively contained the losses to the injection straight with minimal impact on the injection efficiency, at the cost of reducing the stored beam lifetime by $\sim 8 \mathrm{~h}$. The horizontal collimators were observed to begin reducing the injection efficiency at $\pm 12 \mathrm{~mm}$, but losses were not confined to the injection straight until apertures of $\pm 10 \mathrm{~mm}$. The injection efficiency in this case was $\sim 50 \%$. Since the injection efficiency is routinely above $95 \%$, the use of collimators to localise losses is not required. However, they may still be used in the future to provide protection against fault conditions. Closing the IDs does not significantly change this scenario.

02 Synchrotron Light Sources and FELs
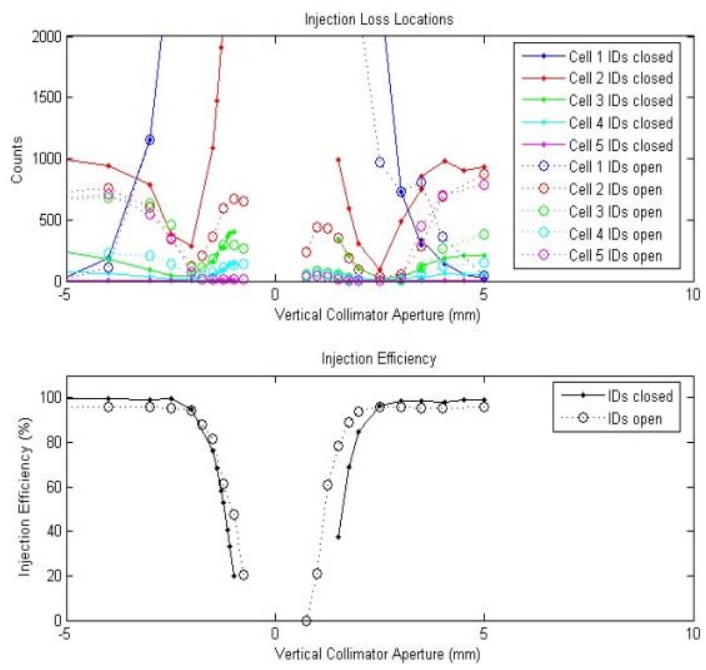

Figure 7: Injection efficiency and beam loss distribution as a function of vertical collimator aperture with/without IDs closed. Vertical collimator apertures of $\pm 2.5 \mathrm{~mm}$ effectively contain losses to the injection straight without impacting upon the injection efficiency.

\section{CONCLUSION}

The Phase I IDs have been commissioned successfully and are running well in User Mode at the design operating point of Diamond's low emittance lattice. Effects on linear optics (linear tunes and beta-beat) are mainly due to the wiggler, as predicted theoretically. Optic perturbations are small and have no effect on beam lifetime. Though the current machine operation does not require any optics compensation, various schemes (feed forward tables for alpha-matching with local and global tunes compensation and the LOCO algorithm) were applied successfully and can be employed whenever required.

The injection efficiency with all IDs closed is normally above $95 \%$ which is a requirement for future top-up operation, although more measurements are required to confirm these results. In-depth investigations of the nonlinear beam dynamics is planned following the installation of "pinger" magnets this summer.

\section{ACKNOWLEDGEMENTS}

It is a pleasure to acknowledge the support and suggestions of the members of the Diamond commissioning team.

\section{REFERENCES}

[1] R. Bartolini et al., these proceedings

[2] A. Baldwin et. al., EPAC 2006, p.3586 (2006).

[3] R. P. Walker, Proc. APAC 2007.

[4] B. Singh et al., EPAC 2006, p. 2092 (2006).

[5] J. Safranek, Nucl. Inst. and Meth. A338, 27, (1997).

[6] I.P.S. Martin et al., these proceedings.

[7] Tracy-II, SOLEIL's version.

[8] P. Elleaume, CERN 90-03, p. 142, (1990). 